\title{
Padma Digestin
}

\section{Tibetisches Multi-Target-Arzneimittel bei dyspeptischen Beschwerden}

Dyspeptische Beschwerden mit Völlegefühl, Druck oder Schmerzen in der Magengegend, Blähungen, Meteorismus und Aufstossen sind häufig und können die Lebensqualität Betroffener stark beeinträchtigen. Sie haben eine multifaktorielle Ätiologie. Es wird vermutet, dass u.a. eine verminderte Peristaltik, übersensibilisierte Schmerzrezeptoren, psychischer Stress, Nahrungsmittelunverträglichkeiten sowie eine Helicobacter-pyloriInfektion beteiligt sein können. Die üblichen therapeutischen Massnahmen wie Lifestyle-Anpassung, Psychotherapie, Protonenpumpeninhibitoren und evtl. Prokinetika erzielen oft kein befriedigendes Ergebnis, weshalb pflanzliche Präparate mit hoher Verträglichkeit und multimodalen Wirkmechanismen immer mehr in den Fokus rücken. Ein solches Phytotherapeutikum ist das Tibetische Arzneimittel Padma Digestin (Swissmedic Nr. 59375).

\section{Padma Digestin: Klinische Wirksamkeit}

Eine klinische Open-Label-Studie am Kantonsspital Liestal zeigte, dass die 6-wöchige Behandlung von Patienten mit funktioneller Dyspepsie (FD) mit Padma Digestin zu einer signifikanten Verbesserung verschiedener dyspeptischer Symptome führte [1]. Dabei reduzierten sich das jeweils als am störendsten empfundene Symptom bezüglich Schweregrad, Frequenz und Beeinträchtigung der täglichen Aktivität sowie u.a. die Symptome postprandiales Völlegefühl, Nausea, Magenkrämpfe, lokalisierter und diffuser epigastrischer Schmerz (Abb. 1). Das hatte eine Verbesserung der Lebensqualität zur Folge, die mittels des PGWBI-S-Fragebogens erfasst wurde. Sie stieg von 55\% auf $70,5 \%$ und erreichte damit fast den Normwert Gesunder $(73,5 \%)$. Im traditionellen Kontext wird die klassische Rezeptur aus Granatapfelsamen und Gewürzkräutern, genannt Granatapfel 5 (tib. Se'bru 5), generell bei geschwächter Verdauungswärme (tib. medrod) angewendet. Eine reduzierte Verdauungswärme hat dabei nicht nur verschiedene dyspeptische Beschwerden zur Folge, medrod ist auch verantwortlich für andere Körperfunktionen im Abdomen, z.B. die Funktion der Urogenitalorgane. Vermindertes medrod kann sich daher auch z.B. in wiederkehrenden Blasenentzündungen, Infertilität, Schmerzen im unteren Rücken oder in einem generellen Kältegefühl äussern.

\section{Wirkmechanismen von Padma Digestin}

Die pflanzlichen Wirkstoffe von Padma Digestin enthalten eine ganze Reihe von sekundären Pflanzenstoffen, die auf verschiedene Aspekte der Verdauung einwirken können (Tab. 1). Eine Modulation der Motilität und Erregbarkeit gastraler und intestinaler glatter Muskelzellen konnte durch eine experimentelle Studie der Universität Bern nachgewiesen werden [2], wobei die

Tab. 1. Chemische Substanzgruppen, die in den Wirkstoffen von Padma Digestin enthalten sind und deren bekannte Effekte im Zusammenhang mit Verdauungsfunktionen stehen

\begin{tabular}{|c|c|c|c|c|c|c|}
\hline $\begin{array}{l}\text { Substanz- } \\
\text { gruppen }\end{array}$ & 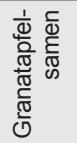 & 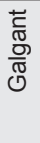 & 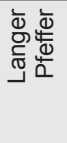 & 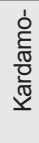 & 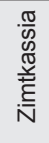 & Bekannte Effekte \\
\hline Ätherische Öle & & $x$ & $\mathrm{x}$ & $x$ & $x$ & $\begin{array}{l}\text { Stimulieren Verdauungssäfte, krampflösend, karminativ, antibak- } \\
\text { teriell, Appetit anregend }\end{array}$ \\
\hline Scharfstoffe & & $x$ & $x$ & & & $\begin{array}{l}\text { Desensitisierung überaktiver Schmerzrezeptoren, stimulieren } \\
\text { Verdauungssäfte, verbessern gastrointestinale Motilität, schleim- } \\
\text { hautschützend, antiemetisch, Appetit anregend }\end{array}$ \\
\hline Flavonoide & & $x$ & & & & $\begin{array}{l}\text { Desensitisierung überaktiver Schmerzrezeptoren, entzündungs- } \\
\text { hemmend, antioxidativ }\end{array}$ \\
\hline Tannine & $x$ & & & & $x$ & $\begin{array}{l}\text { Entzündungshemmend, immunmodulierend, schleimhautschüt- } \\
\text { zend, reizmildernd }\end{array}$ \\
\hline Bitterstoffe & & $x$ & & & & $\begin{array}{l}\text { Stimulieren Magenssäfte, Appetit anregend, verbessern Nährstoff- } \\
\text { aufnahme }\end{array}$ \\
\hline Schleimstoffe & & & & & $x$ & Schleimhautschützend, reizmildernd \\
\hline Fruchtsäuren & $x$ & & & & & $\begin{array}{l}\text { Unterstützen Proteinverdauung, entzündungshemmend, immun- } \\
\text { modulierend }\end{array}$ \\
\hline
\end{tabular}

gezeigten Effekte dafür bekannt sind, dass sie zur Magenentleerung und zur intestinalen $\mathrm{Ab}$ sorption beitragen.

Diese Daten deutet auf einen Multi-TargetWirkmechanismus hin, wie er schon für andere tibetische Vielpflanzenrezepturen gezeigt werden konnte und der besonders bei multifaktoriellen Erkrankungen wie z.B. der FD geeignet ist $[3,4]$. Die Ergebnisse unterstützen den Einsatz von Padma Digestin bei Verdauungsstörungen und funktionellen dyspeptischen Beschwerden. Weiter kann das Präparat auch bei allgemeiner Verdauungsschwäche und bei Appetitmangel, z.B. in der Rekonvaleszenz, eingesetzt werden und könnte in dieser Indikation z.B. auch in der Geriatrie eine Rolle spielen.

\section{Literatur}

1 Meier R et al.: Forsch Komplementmed 2013;20(suppl 2): 2-7.

2 Balsiger BM et al.: J Gastrointest Pharmacol Ther 2013;4 9-15.

3 Schwabl H, Vennos C: Schweiz Z Ganzheitsmed 2006;18: 213-218.

4 Simitz S, Vennos C: Schweiz Z Ganzheitsmed 2015;27: 347-353.

Weitere Informationen bei PADMA AG / www.padma.ch

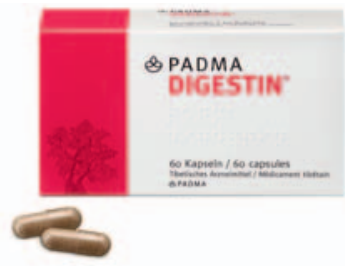

Ausführliche Informationen zu Padma Digestin finden Sie auf www.swissmedicinfo.ch. 


\section{Die Durchblutungsflüsse in unserem Organismus regulieren}

Wie sagte doch Heraklit, der griechische Philosoph: Panta rhei, alles ist im Fluss, in Bewegung. In unserem Organismus herrscht enorme Betriebsamkeit auf allen Ebenen: Auf zellulärer Ebene laufen mehrere Tausend Reaktionen gleichzeitig ab - im Sekundentakt. Mitochondrien produzieren Energie, Muskeln und Nerven kommunizieren über elektrische Impulse und Hormondrüsen sezernieren lebenswichtige Hormone. In Kapillaren, Venen und Arterien läuft die Zirkulation auf Hochtouren.

Damit dieser stetige und lebendige Fluss naturgemäss ungehindert seinen Weg zurücklegen kann, braucht der Organismus gesunde Impulse: Körperliche Bewegung, um die vorgegebenen Mechanismen in Gang zu setzen, eine gesunde, möglichst biologische Ernährung, um den Stoffwechsel aktiv zu unterstützen, und auch von Zeit zu Zeit biologische Medikamente, wenn der Fluss ins Stocken geraten ist.

Dies ist beispielsweise der Fall bei venösen Stauungen bzw. Krampfadern, wenn Venenwandschwäche oder Venenklappeninsuffizienz zum Blutstau, vorwiegend in den Beinvenen, führen.

Die drei Mittel der REGENAPLEX-31erReihe nehmen sich dieses Problems an. Alle drei enthalten die Rosskastanie (Aesculus hippocastanum) und die Zaubernuss (Hamamelis virginiana).

\author{
Speziell in dem REGENAPLEX Nr. $31 b$ \\ wirken folgende Substanzen:
}

Das Thema des Acidum benzoicum in der Homöopathie heisst «Altes beibehalten und auf bessere Zeiten warten», so wirkt es als Simile gegen Stasen und auch entzündungshemmend. Das Acidum hydrofluoricum stärkt, wie allgemein alle Säuren, das Bindegewebe und die Venen und löst Schmerzen bei Krampfadern und entzündeten Narben. Berberis vulgaris und Lycopodium clavatum, beide in der mittleren Potenz D8, wirken indirekt auf die venöse Stase, indem sie primär Entzündungen und Störungen des LeberGalle-Systems behandeln, die sich sekundär durch Blutstauungen und Entzündungen in den Beinen und am After bemerkbar machen. Die Sulfur-Komponenten (Sulfur und Sulfur iodatum), auch hier in mittleren Potenzen, haben einen günstigen Einfluss auf venöse Stauungen, eitrige Entzündungen und Geschwüre der Haut sowie auf akute und chronische Entzündungen.

Es geht aus dieser kurzen Beschreibung hervor, dass das REGENAPLEX Nr. 31b bei Krampfadern besonders gute Dienste leistet, wo die schmerzhafte Blutstauung zur Entzündung neigt, mit allen daraus resultierenden Komplikationen einer Phlebitis oder Thrombophlebitis. Bei ausgeprägten Krampfaderzuständen ist eine langzeitige Therapie mit allen drei Mitteln der 31er-Reihe empfehlenswert. Eine individuelle Betreuung durch einen erfahrenen REGENA-Therapeuten ermöglicht die Wahl des geeigneten Mittels unter Berücksichtigung der Anamnese. Für Therapievorschläge wenden Sie sich an einen erfahrenen Therapeuten aus Ihrer Nähe unter www.regena.ch/ therapeuten-apothekersuche/.

Die Autorin Monique Stahlkopf ist die Begründerin der REGENA-Akademie.

\section{Quelle}

Stahlkopf M: REGENAPLEXE - Natürliche Wirkstoffakkorde. Waiblingen, Status Verlag, 2015 www.status-verlag.de ISBN: 978-3-942924-17-7.

Weitere Informationen bei

REGENA AG

info@regena.ch

www.regena.ch

www.regena-akademie.ch

\section{Teva Pharma lanciert Multiple-Sklerose-Portal}

Auf der neuen Informationsplattform $w w w$. aktiv-mit-ms.ch finden Patientinnen und -Patienten mit Multipler Sklerose (MS) sowie Angehörige Informationen über die Krankheit und den Umgang damit im Alltag. In einem geschützten Bereich, der nur für Fachpersonen zugänglich ist, hat Teva Pharma Servicematerialien und Informationen zum MS-Medikament Copaxone ${ }^{\circledR}$ zusammengestellt, die für die Betreuung von MS-Betroffenen hilfreich sind.

In der Schweiz sind mehr als 10000 Menschen von MS betroffen. Mit der Informationsplattform www.aktiv-mit-ms.ch hat Teva Pharma AG ein Portal geschaffen, das auf alle wichtigen Fragen im Zusammenhang mit der Krankheit und der Therapie eingeht und über Neues aus der Forschung informiert. Für Fachpersonen sind in einem passwortgeschützten Bereich Servicematerialien und wissenschaftlich fundierte Informationen zum Medikament Copaxone aufgeschaltet. Die Website gibt es auch auf Französisch (www.actif-avec-sep.ch).

\section{Unterstützung im Alltag}

Neben der Vermittlung von Wissen stehen Fragen zum Alltag mit MS im Zentrum. Zu den relevanten Themen gehören Familie und Partnerschaft, Freizeit und Reisen, Ernährung und Gesundheit sowie Sport und Psyche. Die zahlreichen Tipps sollen dazu beitragen, die Krankheit besser zu verstehen und den Umgang damit zu erleichtern, sodass trotz MS eine erfüllende Gestaltung des Lebens möglich ist.

\section{Ressourcen durch Training erhalten}

Zusätzlich bietet das Portal Betroffenen die Möglichkeit, ihre kognitiven Fähigkeiten mit- hilfe eines interaktiven Programms, das in Zusammenarbeit mit Spezialisten entwickelt wurde, auf spielerische Weise zu trainieren. Insgesamt stehen vier abwechslungsreiche Lernmodule zur Auswahl, mit denen sich Aufmerksamkeit, Koordination, Merkfähigkeit sowie schlussfolgerndes und räumliches Denken üben lassen.

Weitere Informationen bei TEVA Pharma AG Kirschgartenstrasse 14 4010 Basel, Schweiz office@tevapharma.ch

\section{KARGER}

